\title{
Comment on the editorial relating to: Transversus abdominis plane block compared with wound infiltration for postoperative analgesia following Cesarean delivery: a systematic review and network meta-analysis
}

\author{
Pervez Sultan, MBChB, FRCA, MD (Res) (D) Brendan Carvalho, MBBCh, \\ FRCA $\cdot$ Stephen Halpern, MD, MSc
}

Received: 5 November 2020/ Accepted: 5 November 2020/Published online: 6 January 2021

(C) Canadian Anesthesiologists' Society 2021

\section{To the Editor,}

We are writing on behalf of the authors of our recent article entitled, "Transversus abdominis plane block compared with wound infiltration for postoperative analgesia following Cesarean delivery: a systematic review and network meta-analysis". "We were delighted to have our network meta-analysis selected for publication alongside an accompanying editorial. ${ }^{2}$ The editorial highlights aspects of our study and expertly discusses network meta-analysis methodology. Nevertheless, the editorial reports different interpretations and recommendations compared with the conclusions we drew from our study findings.

The editorial from Drs Barry and Uppal states that there was no difference between single-shot wound infiltration (WI) and inactive controls for the primary outcome of opioid consumption. ${ }^{2}$ While it should be noted that there was no statistical difference between WI and the inactive control group, there still remains the potential for a clinical difference. This can be seen by the $95 \%$ confidence intervals of the standardized mean difference for opioid consumption that we reported as being -3.46 to 0.09 (as outlined in Table 2a and Fig. 3) in favour of a reduction in

This letter is accompanied by a reply. Please see Can J Anesth 2021; this issue.

P. Sultan, MBChB, FRCA, MD (Res) ( $₫) \cdot$ B. Carvalho , MBBCh, FRCA

Department of Anesthesiology, Perioperative and Pain Medicine, Stanford University, Stanford, CA, USA

e-mail: psultan@stanford.edu

S. Halpern, MD, MSc

Department of Anesthesia, Sunnybrook Health Sciences Centre,

University of Toronto, Toronto, ON, Canada opioids with the use of WI. We feel that all active interventions in this study (wound catheters [WC], WI, and transversus abdominis plane [TAP] blocks) are likely to be clinically advantageous compared with inactive controls for the primary outcome of opioid consumption. The probability of being the best intervention $\left(\operatorname{Pr}_{\text {Best }}\right)$ for the primary outcome of opioid consumption shows that the inactive control has a $0 \%$ chance of being the best choice for the patient. So, the main message is that some intervention is likely better than no intervention. Based on the data from this network meta-analysis, implying that WI is not effective would be misleading. We therefore suggest that WI should be offered to women for analgesia after Cesarean delivery in the absence of long acting neuraxial opioids, if TAP blocks or WC are not available options.

The editorial also states that "the network ranking provides a clinically useful recommendation in favour of TAP blocks and WC over WI and inactive controls." ${ }^{2}$ It is important to note that the League table (Table 2a) for the primary outcome of opioid consumption failed to show any differences between TAP, WI, and WC groups. Therefore, the surface under the cumulative ranking curve (SUCRA) and $\operatorname{Pr}_{\text {Best }}$ tables, which were provided, must be interpreted with caution as there were no significant differences between TAP $v s$ WI, TAP $v s$ WC, or WI $v s$ WC groups. ${ }^{3}$ Although TAP is ranked higher than WC for the outcome of opioid consumption, the conclusion that TAP is better than WC is therefore potentially misleading. There are other reasons to be cautious before recommending TAP blocks over the other treatments. Importantly, SUCRA does not consider the magnitude of differences in effects between treatments, and it does not capture the possibility that chance may explain any apparent differences between treatments. Additionally, the data on which the SUCRA 
was calculated was of moderate quality, as it was downgraded for heterogeneity and imprecision. This implies that the relative efficacy of TAP blocks may differ depending upon the clinical setting. For example, institutions may have more experience with WC techniques but limited experience (and success rates) when performing TAP blocks. Furthermore, additional expertise and resources may be required to perform TAP blocks as noted in the editorial.

The editorial also states that "the continuous WC techniques are unlikely to confer enough analgesic benefit over TAP blocks to warrant their use, given the hindrances to patient recovery." 2 The data shows there is no difference between WC and TAP blocks, so either can and should be used to improve analgesia. The use of WCs is a viable option as they are easy to use, require little regional anesthesia expertise for insertion, and are safe with no reports of local anesthetic toxicity with this technique. We do not agree with the editorial authors that WCs hinder patient recovery as they do not cause motor block, do not contraindicate the use of anticoagulants, and are small devices that do not limit patient mobility.
Funding statement None.

Editorial responsibility This submission was handled by Dr. Hilary P. Grocott, Editor-in-Chief, Canadian Journal of Anesthesia.

\section{References}

1. Sultan P, Patel SD, Jadin S, Carvalho B, Halpern SH. Transversus abdominis plane block compared with wound infiltration for postoperative analgesia following cesarean delivery: a systematic review and network meta-analysis. Can J Anesth 2020; DOI: https://doi.org/10.1007/s12630-020-01818-x.

2. Barry G, Uppal V. Management of pain after cesarean delivery without intrathecal morphine: networking for the best answer. Can J Anesth 2020; DOI: https://doi.org/10.1007/s12630-020-01819-w.

3. Mbuagbaw L, Rochwerg B, Jaeschke R, et al. Approaches to interpreting and choosing the best treatments in network metaanalyses. Syst Rev 2017; DOI: https://doi.org/10.1186/s13643017-0473-z.

Publisher's Note Springer Nature remains neutral with regard to jurisdictional claims in published maps and institutional affiliations.

Disclosures None. 